\title{
Dynamics of Skin Vessels Microcirculation Parameters in Rats at the Hypoxia
}

\section{Flyura Alistratova1, Toropova Yana ${ }^{2}$, Nika Bulavinova ${ }^{3}$, and Ekaterina Smirnova ${ }^{1}$}

${ }^{1}$ Saint-Petersburg State Academy of Veterinary Medicine, Saint-Petersburg, Russia

${ }^{2}$ Bioprosthetics and Cardioprotection Research Lab, "V.A. Almazov National Medical Research Center" Ministry of Health of the Russian Federation

${ }^{3}$ Vocational Education Program, Pediatrics Occupation Studies Program, St. Petersburg State Pediatric Medical University, Saint-Petersburg, Russia

\section{Abstract}

The work is devoted to the study of the effect of interval hypoxic training on the state of microcirculation of the skin in rats in the experiment. The study was conducted on white male Wistar rats weighing $70 \mathrm{~g} \pm 10 \%$. In order to study the state of the dynamic characteristics of blood microcirculation, a change in blood perfusion of the tissue was considered. A non-invasive method for quantifying microvascular perfusion was used. The results showed that inhibition of microcirculation up to $50 \%$ was observed in comparison to this value before exposure during the formation of a compensatory reaction of the body to a hypoxic effect on the 7--14 $4^{\text {th }}$ day of the experiment. It was noted that the predominant role in the regulation of the microvasculature belongs to the passive regulation factor of microcirculation which is characterized by the involvement of blood vessels in the microvasculature due to periodic changes in blood volume in the vessel together with an increase in the arithmetic mean perfusion rate. Thus, experimentally modulated hypobaric exposure, changes the volume of blood in arterioles that is determined by a pulse wave.

Keywords: hypoxia, microcirculation skin, rats, laser doppler flowmetry. (c) Flyura Alistratova et al. This article is distributed under the terms of the Creative Commons Attribution License, which permits unrestricted use and redistribution provided that the original author and source are credited.

Selection and Peer-review under the responsibility of the AgroSMART 2019 Conference Committee.

\section{S OPEN ACCESS}

\section{Introduction}

It is known that hypoxia stimulates the strengthening of the functioning of the vessels of the microcirculatory bed of the skin in the study objects. $[7-10,16]$. In the presented experiment the influence of interval hypobaric training on microcirculatory blood flow and mechanisms of regulation of vascular skin tone in male rats was studied $[1,3,5]$. In addition, we investigated the differences in microcirculation at different stages of acclimatization to find out the optimal period for which the skin microcirculation is able to adapt [12-15]. 


\section{Methods and Equipment}

\subsection{Methods}

The study was performed on 30 male Wistar stock rats, weighing $70 \mathrm{~g} \pm 10 \%$ in accordance with the Protocol of the pilot studies approved by the Commission for monitoring and use of laboratory animals of V.A. Almazov National Medical Research Center" Ministry of Health of the Russian Federation. The animals were kept in standard conditions of the vivarium on a full diet, according to the daily nutritional standards for this species of animals, with a standard daily light-dark regime. Animals of the experimental group (group "EG", n=10) were exposed to hypoxia for 21 days, in the mode of 1 hour-1 time per day. The animals were in the cell in threes, always in the same subgroups. Animals of the control group (group CG, $\mathrm{n}=10$ ), similarly experienced, over 21 days out the course "false" hypoxia with the placement of animals in hypobaric chamber was carried, without sealing it, and turning on the pump in mode 1 hour -- 1 time per day. The comparison group was a group of intact animals (group "IG", $n=10$ ). On day $22^{\text {st }}$, the animals of all experimental groups were removed from the experiment by immersion in a $\mathrm{CO}^{2}$ chamber. The analysis of the effect of interval hypobaric training on the microcirculation of the skin was carried out immediately after a session of hypoxia at a temperature of 22 $24{ }^{0} \mathrm{C}$ by noninvasive laser Doppler flowmetric sensing in the conditions of anesthesia of the animal (isoflurane). Registration of parameters was carried out at the following stages of the experiment: before hypoxic exposure -- point 0 , through the 1st, 7th, 14th and 21st days after the first session of hypoxia. The duration of the recording was 360 seconds. The main parameters of microcirculation were recorded (averaged data):

-arithmetic mean of microcirculation ( $\mathrm{M}, \mathrm{pu}$ ), characterizing the average value of perfusion per unit volume of tissue per unit time;

- standard deviation of the amplitude of blood flow oscillations-modulation of blood flow (primarily due to active (endothelial, myogenic and neurogenic mechanisms of regulation of vascular lumen), as well as passive (formed outside the microcirculation pulse wave from the arteries and pulse wave in the venules) factors) or flax, ( $\sigma, \mathrm{pu})$;

- coefficient of variation, characterizing the ratio between the variability of perfusion (flax) and average perfusion ( $\mathrm{M}$ ) in the study area, indicating the percentage contribution of active regulatory factors in the overall modulation of tissue blood flow, (VC, \%), and also the oscillatory process caused by endothelial ( $A E)$, neurogenic ( $A N)$, myogenic $(A M)$ ), respiratory $(R)$, cardiac $(C)$ factors of microcirculation control. 
The obtained parameters were analyzed by wavelet transform. Microvascular tone components were calculated [2]:

- myogenic (tone of metarterioles and precapillary sphincters, $M)$ : $M T=(\sigma \times A D) /(A M$ $\times \mathrm{MCC})$

- neurogenic (tone of precapillary resistive microvessels, $N)$ : NT $=(\sigma \times A D) /(A N \times M C C)$

Normalized values were also calculated to analyze the contribution of each link to blood flow modulation $((\mathrm{A} / 3 \sigma) \times 100 \%)$ and mean perfusion $((\mathrm{A} / \mathrm{M}) \times 100 \%)$.

The anesthetized rats were fixed in the position on the stomach with the limbs withdrawn to the sides. The measurement area was the inner surface of the lower hind limb, the LDF sensor was located at a distance of $2 \mathrm{~mm}$ from the skin surface.

The results were processed using the GraphPad Prism 7 application software package. Median and interquartile range $--25^{\text {th }}$ and $75^{\text {th }}$ percentiles (IU [25\% -75\%]) were indicated to describe features differe from normal distribution. To assess the validity of the differences before and after exposure, the $T$ criterion (Wilcoxon pair criterion) was applied. The $U$ (Wilcoxon-Mann-Whitney) criterion was used to assess the validity of the differences between two non-conjugate populations. $\mathrm{P}<0.05$ was taken as the critical level of statistical significance.

\section{Results}

Initially, the indicators of microcirculation in all groups did not differ statistically ( $p>0.05)$. In the control group "IG" statistically significant changes in the index of microcirculation (IM) for the $21^{\text {st }}$-day interval of exposure to hypoxic training were not revealed, whereas in the control group "CG"-" false hypoxia " of rats tends to decrease microcirculation after $7-14^{\text {ht }}$ days from $10.74[8,38 ; 13,6]$ to $8.75[6,98 ; 10,22]$ pu and the reverse recovery of this indicator to the $21^{\text {st }}$ day to 10,6 pu while there was a statistically unreliable tendency to increase the flax parameter due to increased variability of the VC (flax), with an almost constant value of the microcirculation index.(Fig. 1)

In rats of the experimental group "EG", the initial values of the parameter (IM) were 10.4 [9.19; 14.94] pu, VC 5.8 [5.0; 11.4]; flux (б) 0.5 [0.49; 0.54] pu 14.94] pu, VC 5.8 [5.0; 11.4] \%; flux 0.5 [0.49; 0.54] pu. On the first day, an increase parameter (IM) was observed to 12.95 [9.7; 15.63] pu ( $p<0.05$ ), while VC decreased 4.86 [3.25; 6.18] \%, and the standard deviation (flux) increased and was equal to 0.64 [0.37; 0.97] pu. On days 7th-14th, microcirculation (IM) decreased to 5.28 [4.96; 5.63] pu, with an increase in VC, and decrease in the standard deviation (flux) decreased. On the 21st day, an inverse restoration of the parameter (IM) value to 8.1 was noted $[5.47 ; 11.75]$ units with 


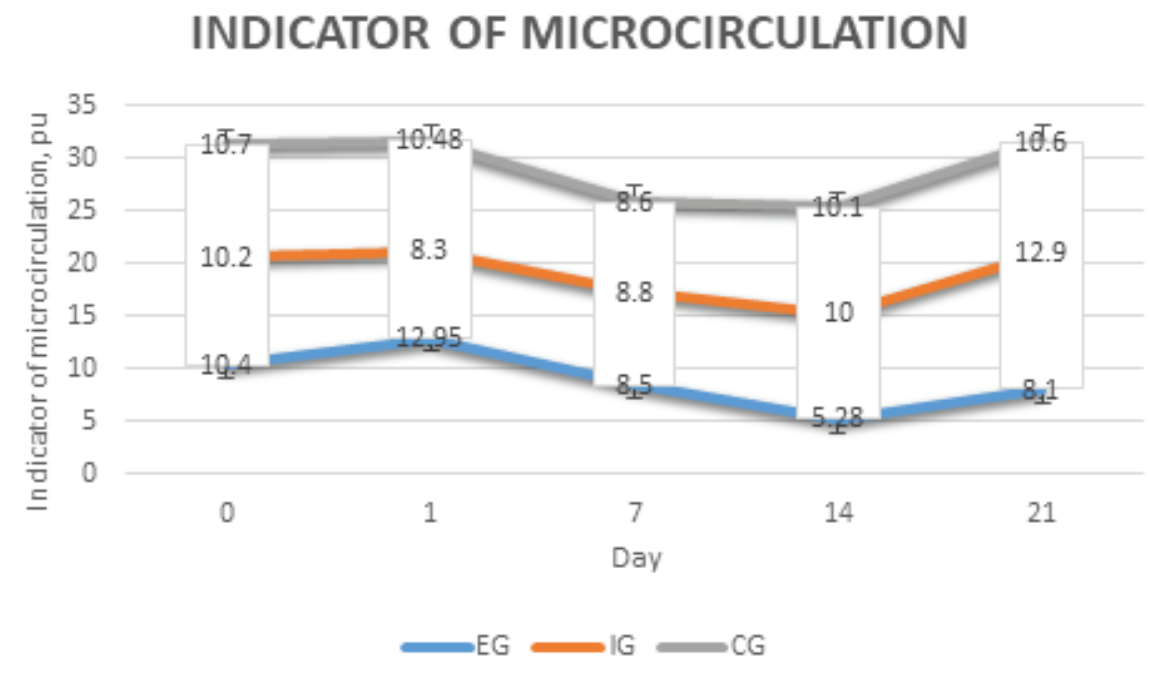

Figure 1: Dynamics of changes in microcirculation during a 21-day course of interval hypobaric exposure.

a simultaneous increase in VC to $5.86[5.17 ; 6.55] \%$ and decrease standard deviation (flux) up to 0.47 [0.31; 0.68] pu (Fig. 2)

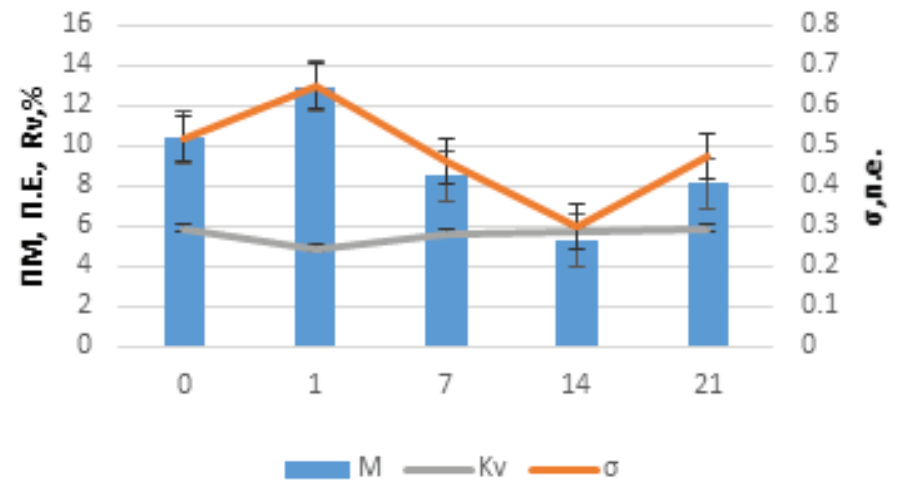

Figure 2: Dynamics of the microcirculation index (IM), the standard deviation of the amplitude of the fluctuation of blood flow $(\sigma)$ and the coefficient of variation of perfusion (VC) at various stages of the experiment, in the EG group.

The revealed changes in the index of microcirculation in rats of the experimental group reflect the change in the reactivity of microvessels. Precapillary that implement the current local regulation of tissue blood flow are characterized by the greatest sensitivity to local extra-and intravasal factors, including the intensity of blood flow. This is due to the stimulating effect of the course of hematopoiesis interval hypobaric training and, as a consequence, an increase in the total number of blood cells after its application. 
Due to the variation of the measurement results, the contribution of active and passive factors of perfusion regulation relative to the mean modulation of blood flow was determined in the group of experimental animals EG [6].

The value of active regulation factors (myogenic) in the EG group decreased on the 1 st $-7^{\text {th }}$ day to $27.7 \%(p \leq 0.05)$, relative to the initial level. On the $14^{h t}-21^{\text {st }}$ day, a reverse increase of this indicator to $50 \%(p \leq 0.05)$ relative to the lowest value $\left(7^{\text {th }}\right.$ days) was noted, which is almost equal to the level of perfusion before exposure [6].

The role of passive microcirculation factors is characterized by a statistically significant decrease in the component associated with the venular link of the respiratory wave. It was noted that on the first day immediately after exposure to a session of hypobaric hypoxia, the parameter decreased by $36.59 \%$. On days $7^{h t}-14^{h t}$, the dynamics of this indicator did not change, the value decreased to $52.2 \%(p \leq 0.05)$ relative to the initial level. Only at 21 days there was an increase of $23.47 \%$ relative to 14 days, which characterizes the recovery processes.

Another passive microcirculation factor, the pulse wave, which reflects the flow of arterial blood into the microvasculature, decreased to $48 \%(\mathrm{p} \leq 0.05)$ on the $1^{\text {st }}-7^{\text {th }}$ day of exposure to the hypoxic factor compared to the initial level. The amplitude of the pulse wave at $14^{\text {ht }}-21^{\text {st }}$ days begins to recover to $46.15 \%(\mathrm{p} \leq 0.05)$, relative to $1^{\text {st }}-7^{\text {th }}$ days of experience.

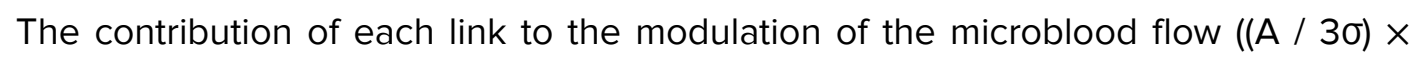
$100 \%)$ was considered. The value of active regulation factors in the $C G$ group before exposure to interval hypobaric hypoxia was 14.2 [10.16; 18.04] cu On days 1--7, there is a statistically unreliable tendency to entrain this parameter to 17.5 [14.56; 19.64$]$ - 15.9 $[14.2 ; 17.73] \mathrm{cu}$. On the 14th day of exposure to hypobaric factor there was an increase in the indicator to 22.9 [17.54; 28.7] cu., and on the 21st day of hypoxic exposure, this parameter was normalized to the initial level of $18.2[14.26 ; 22.56] \mathrm{cu}$. In parallel, in the group of intact rats "IG" there was no change in the value of this parameter, it remained at the same level. In the "EG" experiment group, the initial value of the parameter decreased on the 1st-7th day to $27.7 \%$, on the 14 th day there was a jump in the value of this parameter by $50 \%$, and on the 21 st day there was a reverse decrease of this indicator by $25 \%$ relative to the initial values [6].

An analysis of the role of passive microcirculation factors, reflecting blood flow fluctuations relative to the average microcirculation value, during the course of interval hypobaric exposure revealed a statistically significant decrease in the component associated with the venular link of the respiratory wave by $38.7 \%$ on the first day immediately after exposure to a hypobaric hypoxia session, then the restoration of the value was 


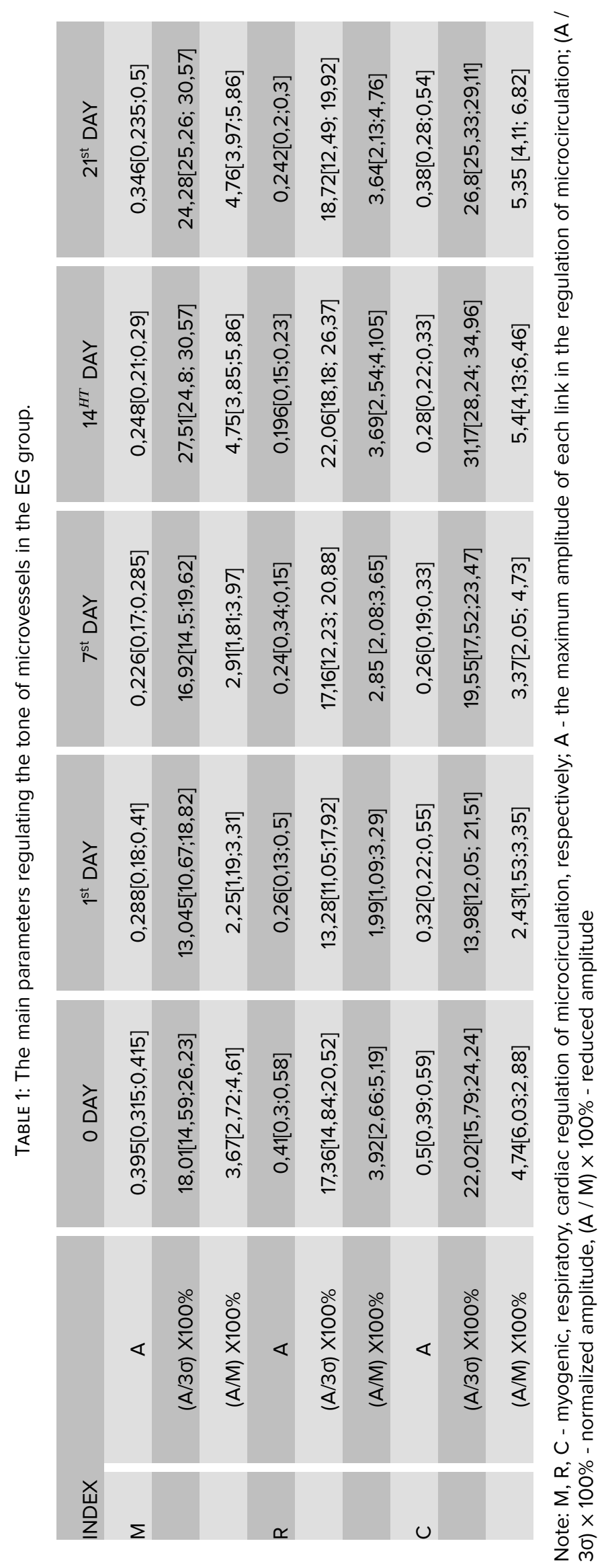


observed of this parameter on the 7th day, to the initial level of 17, $1 \mathrm{cu}[14.14 ; 19.78]$ and the subsequent short-term increase on the 14 th day by $27 \%$ relative to the initial level, and the restoration of this value on the $21^{\text {st }}$ day $-18,72 \mathrm{cu}$ [13.46; 24.8]. In the control group "IG", the value of the studied parameter was kept within the normal range; no significant differences between the values were found. In the group of "false hypoxic" rats - "CG" there is a short-term increase in the respiratory component on the first day to $22.5 \mathrm{cu}$ [15.32; 29.82], after which this value is equalized to the initial level and remains unchanged until the end of the study.

Another passive microcirculation factor, the pulse wave, which reflects the flow of arterial blood into the microvasculature, increased in the EG group by $3 \%$ when exposed to hypoxic factor compared with the IG group. In the experimental group of rats, the amplitude of the pulse wave decreased by $37 \%$ on the first day, after which there was an increase in this indicator by $7^{h t}-14^{h t}$ days to $40 \%$, on the $21^{\text {st }}$ day there was a tendency to decrease the studied parameter by $10 \%$. In the control group "IG" in animals, the dynamics of the respiratory component remains at the same level, at the moment when in the control group "CG" there is a tendency to decrease this parameter, on the first day by $17 \%$ relative to the initial value, with a further return to the initial value on the $7^{\text {th }}$ day and remained at the same level until the last day of the study.

\section{Discussion}

Under the conditions of the simulated effect of hypobaric hypoxia in the EG group of experimental animals, an increase in perfusion of $25 \%$ was observed on the 1st day of hypoxic exposure, with an increase in the coefficient of variation and a decrease in the flux parameter, which may be associated with an urgent compensatory increase in the content of red blood cells, blood flow from the depot. At the same time, a decrease in the proportion of respiratory and cardiac components of vascular tone is observed. This effect may be due, inter alia, to the effect of this factor on smooth muscle precapillary, since the indicator of myogenic tone during this period remains within the standard values. In the control group "CG" there is also a statistically insignificant increase in perfusion, while in the control group "IG" this value is kept within the normal range. However, in the process of development of an adaptive compensatory reaction in the EG group of experimental animals, a decrease $(p \leq 0.05)$ in the level of perfusion was observed on days $14^{h t}-21^{\text {st }}$, due to the outflow of circulating blood into the vessels of the central bloodstream. In the same period, this value in animals of the control groups "IG", "CG" has a slight tendency to increase. 
It was proved that hypoxic effects affect systemic changes in inflammation. In the presented study, on the model of septic shock in animals, the parameters of the microvasculature were studied using intravital microscopy, which correlated with oxygen metabolism. The fact that an increase in the rate of oxygen extraction cannot attenuated that microcirculation sufficiently, is has been fully studied [18].

A study of the functioning of regulatory systems of peripheral blood flow led to the following results. In the EG group of the experimental animals, there were no significant changes in vibration amplitudes in the ranges of myogenic, endothelial and neurogenic activity relative to the CG control group, in the course of $21^{\text {st }}$-day hypobaric training. In the control group "IG" during the three-week hypobaric course also no significant changes in the corresponding parameters were detected relative to the control group "CG".

However, during the development of acclimatization to hypobaric effects in animals of the EG group, the amplitudes of blood flow fluctuations in the range of respiratory and cardiac activity significantly increased on the $14^{h t}-21^{\text {st }}$ days of the study by $30 \%$ ( $p$ $<0.05)$ and $40,9 \%(p<0.05)$, respectively, compared with the corresponding indicators in the control groups of subjects "CG", "IG".

In experimental animals, "EG" showed an increase in skin blood perfusion on the 1 st day relative to control groups of animals during hypobaric training. On the $14^{h t}-21^{\mathrm{st}}$ day, the reverse dynamics of a decrease in blood perfusion index of blood vessels was indicated, while in the two control groups "CG", "IG" no significant changes were found, moreover, there was a tendency to increase this parameter. In the "EG" group of experimental subjects, in the process of establishing the acclimatization of blood vessels of the microvasculature of hyperemia, an increase in the amplitudes of fluctuations in blood flow in the cardiac and respiratory ranges was revealed.

The above changes in microcirculation parameters in animals of the experimental group "EG" may be associated with local changes in the functioning of the vascular components of the skin, which stimulates adaptive changes in peripheral hemodynamics. These changes can be considered systemic, presumably associated both with stimulation of the processes of microangiogenesis, and optimization of the rheological characteristics of the blood.

\section{Conclusion}

Thus, the use of a course of interval hypobaric hypoxia in rats causes a change in all indicators of microcirculation with a predominance of factors regulating microcirculation 
of the skin. The results showed that a course of interval hypobaric training leads to inhibition of microcirculation on the $7^{\text {th }}$ and the $14^{\text {th }}$ days, due to compensatory outflow of blood to the central circulatory system and its partial recovery on the $21^{\text {st }}$ day. According to the wavelet analysis of the contribution of various factors to the modulation of blood flow, an increase in the proportion of passive stimulation factors by $34 \%$ (pulse wave) of microcirculation was noted. Thus there is an active flow of blood into the microvasculature modulated by a pulse wave. Improviment of the state of microcirculation on the $21^{\text {st }}$ does indicates a positive adaptive dynamic after a course of interval hypoxic training.

\section{Acknowledgement}

The authors would like to express gratitude to their colleague for their contribution and support to the research. They are also grateful to all the reviewers who gave their valuable inputs to the manuscript and helped in completing the paper.

\section{Conflict of Interest}

The authors have no conflict of interest to declare.

\section{References}

[1] Enikeev, D.A., Ryakhovsky, A.E., Fatkullin, K.V. et al. (2015). The dynamics of peripheral blood flow in rats with acute carbon monoxide poisoning according to laser Doppler flowmetry Modern problems.

[2] Krupatkin, A.I. (2014). Blood flow oscillations -- new diagnostic language in microvascular research. Regional hemodynamics and microcirculation, vol. 13(1), pp. 83--99.

[3] Félétou, M., Köhler, R., Vanhoutte, P.M. (2012). Nitric oxide: orchestrator of endothelium-dependent responses. Ann Med., vol. 44, no. 7, pp. 694--716.

[4] Serebrovskaya, T.V., Shatilo, V.B. (2014). The experience of using interval hypoxia for the prevention and treatment of diseases of the cardiovascular system. Overview. Zhurn. blood circulation and hemostasis, vol. 1--2, pp. 16--33

[5] Solovyova, A. G., Krylova E. V., Nepryakhina, I. I., Peretyagin, P. V. (2015). The state of microcirculation in rats with thermal injury under the influence of royal jelly. Bioradicals and antioxidants, vol. 2, no. 4, pp. 50--55. 
[6] Shi, R., Chen, M., Tuchin, V.V. et al. (2015). Accessing to arteriovenous blood flow dynamics response using combined laser speckle contrast imaging and skin optical clearing, Biomed. Opt. Express, vol. 6(6), pp. 1977--1989.

[7] Jung, C., Jung, F., Kelm, M. (2016). The microcirculation in hypoxia: The center of the battlefield for oxygen Clinical Hemorheology and Microcirculation, vol. 63, no. 3, pp. 169--172.

[8] Benedikt, T., Kleinsasser A., Stadlbauer K. et al. (2018). Cutaneous Microvascular Blood Flow and Reactivity in Hypoxia Frontiers in Physiology, vol. 9 pp. 154--157.

[9] Paparde, A., Plakane, L., Circenis, K., Aivars, J.I. (2015). Effect of acute systemic hypoxia on human cutaneous microcirculation and endothelial, sympathetic and myogenic activity. Microvasc. Res. no. 102, pp. 1--5.

[10] Niedermeier, M., Weisleitner, A., Lamm, C., Ledochowski, L., Frühauf, A., Wille, M. et al. (2017b). Is decision making in hypoxia affected by pre-acclimatisation? A randomized controlled trial. Physiol. Behav, no. 173, pp. 236--242.

[11] Casey, D.P., Joyner, M.J. (2012). Compensatory vasodilatation during hypoxic exercise: mechanisms responsible for matching oxygen supply to demand. J. Physiol., no. 590, pp. 6321--6326.

[12] Baboshina, N.V. (2016). Evaluation of microcirculation in children of 8 and 10 years of age using inspiratory breath hold. Bulletin of Russian State Medical University, no. 3, pp. 51--56.

[13] Feng, W., Zhang, Ch., Yu, T., Zhu, D. (2019). Quantitative evaluation of skin disorders in type 1 diabetic mice by in vivo optical imaging, Biomed. Opt. Express, vol. 10, pp. 2996--3008

[14] Feng, W., Shi, R., Zhang, Ch. et al. (2017). Lookup-table-based inverse model for mapping oxygen concentration of cutaneous microvessels using hyperspectral imaging. Opt. Express, vol. 25, pp. 3481--3495.

[15] Arutyunyan, G.A., Aliyev, A.R., Vlasov, K.E. et. al. (2018). Arterial infusion in the treatment of patients with acute pancreatitis. Bulletin of Medical Science, no. 2(10), pp. 52--55.

[16] Popovich, N.V., Raspopova, E.A. (2018). The role of breath holding at the initial stage of teaching children with mental disorders swimming. The Russian Journal of Physical Education and Sport., no. 13(4), pp. 39--42.

[17] Willis, S.J., Peyrard, Rupp, A.T. et al. (2019). Vascular and oxygenation responses of local ischemia and systemic hypoxia during arm cycling repeated sprints. Journal of Science and Medicine in Sport. DOI: 10.1016/j.jsams.2019.05.001. 
[18] An X., Zhang H., Sun Y., and Ma X., The microcirculatory failure could not weaken the increase of systematic oxygen extraction rate in septic shock: An observational study in canine models. Clin Hemorheol Microcirc 63 (3) (2016), 267--279. DOI 10.3233 / $\mathrm{CH}-152022$ 